cells and the CD33 +CD11b+myeloid derived suppressor cells were assessed using flow cytometry.

Results: After 20 weeks of exercise intervention there was a decrease in the frequency of Foxp3 +CD25+CD127 regulatory T cells and CD24hiCD38hi B cells but no change was observed in the active control group. The reduction in Tregs by exercise was most pronounced in the female participants. Despite lower levels of adaptive immune cell populations the disease activity did not increase.

Conclusions: Aerobic and resistance exercise in elderly patients with rheumatoid arthritis lead to a decreased number of regulatory Foxp3 +CD25+CD127 reggulatory $T$ cells and CD24hiCD38hi B cells. This decrease was not associated with an increased disease activity score or increased inflammation.

Disclosure of Interest: None declared

DOI: 10.1136/annrheumdis-2018-eular.4668

\section{FRI0085 A STUDY OF THE RELATIONSHIP BETWEEN SERUM VITAMIN D LEVEL AND DISEASE ACTIVITY IN RHEUMATOID ARTHRITIS PATIENTS}

S. Datta ${ }^{1}$, A. Kar ${ }^{2}$, T.K. Bhaumik ${ }^{3}$, T. Santra ${ }^{4} .{ }^{1}$ Anti Retroviral Therapy Center, ${ }^{2}$ Department of General Medicine, Malda Medical College and Hospital, Malda; ${ }^{3}$ Department of General Medicine, Calcutta Medical Research Institute, Kolkata; ${ }^{4}$ Department of General Medicine, Midnapore Medical College and Hospital, Midnapore, India

Background: Vitamin D is an essential component of our body. Approximately $3 \%$ of the human genome is directly or indirectly regulated by the Vitamin D endocrine system, which supports the idea that Vitamin D insufficiency has widespread adverse consequences for human health. ${ }^{[1]}$ Till date several studies have been done regarding the relation of Vitamin $\mathrm{D}$ and Rheumatoid Arthritis but there are limited studies in Eastern India. Hence this study is being done to examine the relation of serum 25 hydroxy Vitamin D level and Disease Activity in Rheumatoid Arthritis patients.

Objectives:

- To examine the relationship between Serum 25 Hydroxy Vitamin D level and SDAI (Simplified Disease Activity Index) in Rheumatoid Arthritis patients.

- To examine the relationship between Serum 25 Hydroxy Vitamin D level and Tender Joint Count(TJC), Swollen Joint count(SJC) and CRP in

Rheumatoid Arthritis patients.

- To evaluate the relation of serum vitamin D level with various socio demographic factors like Gender, Diet, Occupation, Season, Height, Weight, BMI in Rheumatoid Arthritis Patients.

Methods: Ninety six RA patients attending The Rheumatology clinic of Out Patient Department of An Urban Tertiary care hospital (Latitude of KOLKATA is $22^{0} 32 \mathrm{~N}$ ) from October 2013 to September 2014, fulfilling the ACR -EULAR 2010 criteria for classification of RA, were included in the study. $25(\mathrm{OH})$ vitamin $D$ levels were measured. Disease activity of RA was assessed by SDAl score.

Results: More than Ninety Percent of the RA patients were found to have either Vitamin $D$ deficiency or insufficiency. The mean serum vitamin $D$ level of these RA patients was $20.02( \pm 8.92) \mathrm{ng} / \mathrm{ml}$. The RA patients with High Disease Activity (SDAl between 26.1 and 86 ) had significantly low $(p<0.0001)$ mean serum vitamin D level $[11.11( \pm 6.08) \mathrm{ng} / \mathrm{ml}]$ than those with Moderate (SDAl between 11.1 and 26) or Low Disease Activity (SDAl between 3.4 and 11.0) whose serum vitamin D level was $21.15( \pm 7.47) \mathrm{ng} / \mathrm{ml}$ and $25.58( \pm 7.30) \mathrm{ng} / \mathrm{ml}$ respectively. There is a significant negative correlation between the Serum Vitamin D level and SDAI score $(r=-0.669, p<0.0001)$ in the whole group of the study population. However On analysing the data separately in RA patients with Vitamin D deficiency, insufficiency and sufficiency, this significant relation is separately evident only in the RA patients with Vitamin D deficiency (serum vitamin D level $<20 \mathrm{ng} / \mathrm{ml}$ ) but not in those who were in the insufficient or sufficient groups. There is an independent negative impact of Simplified Disease Activity Index (SDAI) on Serum Vitamin D

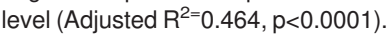

Conclusions: RA patients having high disease activity in terms of SDAI Score had significantly low vitamin D level compared to patients of RA having low or moderate disease activity. Lower levels of serum vitamin D was associated with increased disease activity in RA patients. On subgroup analysis, there is significant negative correlation separately evident only in the RA patients with Vitamin D deficiency (serum vitamin $D$ level $<20 \mathrm{ng} / \mathrm{ml}$ ) but not in those who were in the insufficient or sufficient groups.

\section{REFERENCE:}

[1] Bouillon R, Carmeliet G, Verlinden L, et al. Vitamin D and human health: lessons from vitamin D receptor null mice. Endocr Rev 2008;29:726-776.

Disclosure of Interest: None declared

DOI: 10.1136/annrheumdis-2018-eular.1233

\section{FRI0086}

IS THE DISCORDANCE BETWEEN THE DOCTOR AND THE PATIENT A DETERMINANT OF ADHERENCE?

T. Oton ${ }^{1}$, J. Calvo-Alén ${ }^{2}$, L. Cea-Calvo ${ }^{3}$, L. Carmona' . 'INMUSC, Madrid;

${ }^{2}$ Hospital Universitario Araba, Vitoria; ${ }^{3}$ Medical Affairs, Merck Sharp and Dohme S. A, Madrid, Spain

Background: Adherence is a critical factor in the therapeutic response in rheu matoid arthritis (RA), which may be influenced by the doctor-patient relationship. In the ARCO study, we previously reported a percentage of lack of adherence to the subcutaneous biologic of $14.3 \%$ during the first 14 months of treatment, and that the adherence was better in patients without induction and with a monthly administration schedule. ${ }^{1}$ In this post hoc analysis, we explored whether doctorpatient disagreements may be related to lower adherence rates.

Objectives: To analyse the percentage of patients with discrepancies in the evaluation of the activity of the disease between doctors and patients and a possible association between the existence of disagreement and adherence to subcutaneous biological drugs.

Methods: The ARCO study was a multicenter, cross-sectional study in which patients with RA were included according to EULAR-ACR 2010 criteria, who had been prescribed a subcutaneous biological drug in the previous 12-18 months. As part of the evaluation of the disease, patients and doctors were asked to rate the disease on a visual analogue scale (VAS), with values ranging from 0 to 10 with higher values indicating worst symptoms. Disagreement was defined as a difference of $>/=3$ points between the absolute values. Adherence was assessed retrospectively by means of the Medication Posession Ratio (MPR), considering adherence those patients with MPR $>80 \%$. The association between adherence and disagreement was studied using bi and multivariate logistic regression models with covariates-adjustments.

Results: We included 360 patients ( $77.5 \%$ women, mean age: $55 \pm 0.6$ years). Disagreement was detected in $56(15.5 \%)$. In patients with disagreement, the mean VAS score of the patient was $5.75 \pm 1.8$ versus $2.7 \pm 2.2$ in the group without disagreement $(p<0.001)$, and there were no differences in terms of the doctors VAS (group with disagreement $=2.7 \pm 1.8$ versus $2.2 \pm 2.0$ in the group without dis agreement, $p=0.110$ ). The two groups of patients presented differences in terms of age ( 5 years more than average in the group with disagreement, $p=0.010$ ) presence of comorbidity (14\% more frequent in the group with disagreement, $\mathrm{p}=0.030)$ and the value of the mean DAS28 ( 0.6 points higher in the group with disagreement, $\mathrm{p}<0.001)$. Among the patients who presented a VAS disagreement, the percentage of non-adherence was $10.7 \%$, and of $14.5 \%$ among those who had a VAS similar to the doctor $(p=0.45)$. The regression analysis showed no difference in the association between adherence and disagreement; by introducing into the models covariates associated with adherence (induction, frequency of administration and age) or with disagreement (age, comorbidity and DAS28).

Conclusions: We observed a disagreement between patients and doctor VAS scores in $15.5 \%$ of cases, with higher values coming from patients. We did not observed an association between this disagreement and adherence to subcutaneous biological drugs.

\section{REFERENCE:}

[1] Calvo-Alen J, Monteagudo I, Salvador G, Vazquez-Rodriguez TR, TovarBeltran JV, Vela $P$, et al. Non-adherence to subcutaneous biological medication in patients with rheumatoid arthritis: a multicentre, non-interventional study. Clin Exp Rheumatol 2017;35(3):423-30.

Acknowledgements: This is a secondary analysis of the ARCO study, which was financed by Merck Sharp and Dohme Spain.

Disclosure of Interest: T. Oton: None declared, J. Calvo-Alén : None declared, L. Cea-Calvo Employee of: Merck Sharp and Dohme S.A, L. Carmona: None declared

DOI: 10.1136/annrheumdis-2018-eular.6668

\section{FRI0087 REGIONAL AGE- AND SEX-SPECIFIC INTIMA-MEDIA THICKNESS CRITERIA IN YOUNG PATIENTS WITH RHEUMATOID ARTHRITIS}

V. Omelchenko ${ }^{1,2}$, E. Letyagina ${ }^{1}$, M. Korolev ${ }^{1}$, R. Khapaev ${ }^{1}$, T. Pospelova ${ }^{2}$, V. Konenkov ${ }^{1} .{ }^{1}$ FSBSI «Scientific Institute of clinical and experimental lym»hology» - a branch of the FSBSI "Federal Research Center of the Institute of Cytology and Genetics of the Siberian Branch of the Russian Academy of Sciences";

${ }^{2}$ Novosibirsk State Medical University, Novosibirsk, Russian Federation

Background: Rheumatoid arthritis (RA) is associated with early progression of cardiovascular (CV) diseases. The $\mathrm{CV}$ risk calculators had been verified on a specific population ${ }^{1}$ and should be applied predominantly for them. Systematic COronary Risk Evaluation (SCORE) was recommended by EULAR for use in RA patients with multiplying coefficient $1,5{ }^{2}$ Nevertheless, SCORE don't consider RA-specific factors and couldn't be used in young patient. For timely prevention 
additional criteria is needed to evaluate $\mathrm{CV}$ risk in RA-patents. A presence of atherosclerotic plaque (API) or intima-media thickening, assessed by carotid ultrasonography, may be used as a high CV risk marker after adjustment by age and sex factors.

Objectives: To investigate the prevalence of carotid intima-media thickening, using regional age- and sex-specific criteria.

Methods: One hundred forty eight Caucasian patients with RA (age 53 years; ${ }^{40 ;} 60$ DAS28 5.01 [3.91; 5.90]) without (API) were included in our study. Patients had ACR-defined RA (1987 classification criteria). All patients gave written informed consent before enrollment. SCORE with multiplying coefficient 1.5 was used for the CV risk determining. Range of atherosclerotic progression was assessed by ultrasonography with measurement of carotid intima-media thickness (IMT). IMT measured had been compared with ranges followed:<35/35-44/ 45-54/55-64/65-74/>75 age - 0,55/0,72/0,72/0,80/0,86/1,0 $\mathrm{mm}$ for the right artery and $0,53 / 0,65 / 0,73 / 0,85 / 0,89 / 1,0 \mathrm{~mm}$ for the left one (men); 0,47/0,51/0,71/ $0,78 / 0,87 / 0,87 / 0,87 \mathrm{~mm}$ for the right artery and $0,50 / 0,55 / 0,71 / 0,80 / 0,91 / 0,98$ for the left one (women), respectively. ${ }^{3}$ Descriptive statistics, Chi-squared test, Spearman rank correlation coefficient were used for data analysis. Results are presented as median and 25th/75th percentiles (Me [25th percentile; 75th percentile].

Results: IMT significantly correlated with age $(\rho=0,63 ; \mathrm{p}<0,001)$, systolic blood pressure $(\rho=0,22 ; \boldsymbol{p}=0,017)$, but not with other parameters (sex, smoking, cholesterol, etc). Risk was evaluated by SCORE for 109 RA patients elder than 40 years (age -57 years $^{50 ; 63}$ ) and was $0 \%>15 \%(1.95 \%$ [0.75; 3.15]). An intima-media thickening had been revealed in 86 from 109 (78,9\%) patients and correlated with SCORE value $(\rho=0,42 ; p<0,001) .34$ patients $(23.0 \%)$ were younger than 40 years (age -30.5 years ${ }^{28,0}, 36,0$ ), therefore the relative $C V$ risk scale had been used for ones. Risk evaluated was medium (1.5\%-3\%) for all young patient. An intima-media thickening had been revealed in 31 from $34(91.2 \%)$ patients and didn't correlate with SCORE value $(\rho=0.37 ; p=0.104)$. Number of patients with carotid thickening between two groups didn't significantly difference $(p=0.13)$.

Conclusions: Using age- and sex-specific criteria for the IMT evaluation may be useful in young patients with RA.

\section{REFERENCES:}

[1] Conroy RM, et al. Estimation of ten-year risk of fatal cardiovascular disease in Europe: The SCORE project. Eur. Heart J. 2003;24(11):987-1003.

[2] Lunzer R. 2016 updated EULAR evidence-based recommendations for the management of gout. J. fur Miner 2016;23(3):108.

[3] Malyutina S. K., et al. Sex- and age-specific reference criteria of carotid arteries intima-media thickness in typically Russian population. Ultrasonic and functional diagnostics 2015;(5S):107b.

Disclosure of Interest: None declared DOI: 10.1136/annrheumdis-2018-eular.6089

\section{FRI0088 PSYCHOLOGICAL IMPACT IN RHEUMATOID ARTHRITIS: ROLE OF SPECIFIC TREATMENT AND ASSOCIATED MEASURES}

X. Grapton ${ }^{1}$, on behalf of CREER, P. Lemesle ${ }^{2}$, L. Arabian ${ }^{3}$, on behalf of CREER, V. Strotz ${ }^{4}$, on behalf of CREER. ${ }^{1}$ Private Rheumatology Practice, Colombes; ${ }^{2}$ Private Rheumatology Practice, Bois-Colombes; ${ }^{3}$ Private Rheumatology Practice, Clamart; ${ }^{4}$ Private Rheumatology Practice, Antony, France

Background: Psychological issues in rheumatoid arthritis (RA) are often spontanously mentioned by patients or identified by rheumatologists. Besides classic follow up parameters like DAS28, we have to consider those issues to improve our patients psychological well-being

Objectives: Explore reasons of psychological impact in RA. Analyse in a quantitative way symptoms of discontent and their repercussions. Evaluate consequences of disease treatment on those symptoms and propose recommendations for rheumatologists.

Methods: RA cases were collected by a group of 20 private practice rheumatologists in the Paris area. Basic informations about the patient and his disease were provided by his rheumatologist. Questionnaire including 14 items about psychological and life quality involvement was filled in by the patient.

Results: 167 cases collected : $82 \%$ women, mean age 57 years, $56 \%$ moderate and $14 \%$ severe disease, $76 \%$ ACPA positive, $73 \%$ structural damage. Initial DAS28 4,7. Post treatment DAS28 2.7. Drugs: classic DMARDs 95\%, corticosteroids $73 \%$, biological DMARDs $22 \%$, combination therapy $76 \%$.
In $82 \%$ of the cases, the rheumatologist recommends more than only specific RA drug treatment: rest (47\%), physiotherapy, ergotherapy, balneotherapy (42\%), adapting professionnel activity and environment, help for housework (33\%), psychological support or psychoactive drugs (19\%), yoga or other relaxation exercices $(11 \%)$, balneology..

Psychological repercussions are spontaneously mentioned by $71 \%$ of the patients.

Psychological state is altered by : disease announcement, pain $(85 \%)$, physical fatigue $(71 \%)$, handicap $(58 \%)$, other people's attitude.

Are limited : housework (51\%), getting about (44\%), do-it-yourself $(55 \%)$, sports $(61 \%)$, artistic activities (41\%), professional activity (29\%).

Social life is affected by : lack of being listened to $(72 \%)$, lack of being understood (68\%), lack of empathy (49\%).

RA treatment reduces all those factors by at least one third, except impact on family relations.

Before treatment, RA patients report: sleeping trouble (70\%), anxiety (57\%), lack of motivation $(55 \%)$, dependency $(49 \%)$, frustration $(42 \%)$, lack of self-esteem $(37 \%)$, concentration problems $(35 \%)$, disillusion $(31 \%)$, depression $(30 \%)$, family tension (29\%), isolation (27\%) and libido decrease (23\%).

After treatment, all those factors are $41 \%-55 \%$ less frequent, except libido decrease $(-28 \%)$.

$55 \%$ sleep better, $49 \%$ aren't depressed anymore, concentration problems diminish by $48 \%$ and social life is improved by $50 \%$.

Conclusions: There is a major psychological impact in RA patients, with repercussions on their well-being and life quality. This can be improved by specific ant RA treatment, but we must also consider other measures and, if necessary, psychological support and/or psychoactive drugs. All chosen items are improved, except impact on family relations.

Disclosure of Interest: None declared

DOI: 10.1136/annrheumdis-2018-eular.2277

\section{FRI0089 ASSESSMENT OF COGNITIVE FUNCTION IN RHEUMATOID ARTHRITIS}

Z. Kardos $^{1}$, C. Oláh ${ }^{2}$, M. Andrejkovics ${ }^{3}$, E. Bodnár ${ }^{2}$, K. Hodosi $^{4}$, K. Fazekas ${ }^{1}$, Á. Flórián ${ }^{1}$, K. Lukács ${ }^{1}$, Á. Miksi ${ }^{1}$, Z. Baráth ${ }^{1}$, L. Tamási ${ }^{1}$, Z. Szekanecz ${ }^{4}$ ${ }^{1}$ Department of Rheumatology, Semmelweis Teaching Hospital; ${ }^{2}$ Department of Neurosurgery, Borsod County Teaching Hospital, Miskolc; ${ }^{3}$ Institute of Behavioural Sciences, University of Debrecen, Faculty of Public Health; ${ }^{4}$ Department of Rheumatology, University of Debrecen, Faculty of Medicine, Debrecen, Hungary

Background: For long, rheumatoid arthritis (RA) was thought not to associate with central nervous system (CNS) involvement. In recent years some studies suggest that cognitive function is impaired in RA patients. Accelerated atherosclerosis and reduced function of intracranial vessels in RA can be associated with vascular dementia.

Objectives: We assessed RA patients and healthy controls by neuropsychological tests, cognitive function such as attention, intelligence, memory tests and also by anxiety and depression tests. We wished to explore the prevalence of neuropsychiatric manifestations and cognitive impairment in patients with RA. Intracerebral vascular lesions were investigated by brain MRI.

Methods: Sixty RA female patients were included. Among them, 20 were MTXtreated, biologic-free, 40 patients received biologics. The controls included 39 non-RA healthy women. The following standardised tests were used: the Montreal Cognitive Assesment (MoCa) Test, the Victoria Stroop Test (VST), the Spielberger State-Trait Anxiety Inventory (STAI), the Benton Visual Retention Test (BVRT), the Beck Depression Inventory (BDI), the Brigg's and Nebe's Test, the Trail Making Test (TMT) A and B, the Word fluency with the letter (WF-L) and with category naming tests (WF-C), the Rey-Osterreich Auditory Verbal Learning Test (RAVLT), the Weschler Adult Intelligence Scale (WAIS). We also performed brain $\mathrm{MRI}$ in all patients in order to associate cognitive function with MRI changes.

Results: The MoCa total score was significantly lower in RA patients $(23.3 \pm 3.8)$ especially in biologic-treated group $(22.6 \pm 4.3)$ compared to controls $(25.6 \pm 2.4)$ $(p=0,002 ; 0,001)$. The attention MoCa test score was significantly lower in biologic- $(4.5 \pm 1.6)$ compared to MTX-treated patients $(5.7 \pm 0.6)(p=0.001)$. The STA scores were significantly higher in RA (STAIS: $45.5 \pm 8.5$; STAIT: $48.0 \pm 11.0$ ) compared to controls (STAIS: $36.9 \pm 9.1$; STAIT: $41.1 \pm 9.0)(p<0.001 ; 0.002)$. The BD score was significantly higher in RA $(13.2 \pm 8.8)$ and in biologic-treated patients $(13.7 \pm 8.7)$ than in controls $(8.9 \pm 6.5)(p<0.05)$. The TMT scores were significantly higher in RA (TMT-A: 69.0 \pm 26.3 ; TMT-B:100.2 \pm 48.5 ) compared to controls (TMTA: $53.1 \pm 14.3$; TMT-B: $53.1 \pm 22.7)(p<0,05)$. The VST scores were also significantly higher in RA vs controls. The WAIS and Benton scores were significantly lower in 